\title{
NON-DEGENERACY AND UNIQUENESS OF SOLUTIONS TO SINGULAR MEAN FIELD EQUATIONS ON BOUNDED DOMAINS
}

\author{
DANIELE BARTOLUCCI, ALEKS JEVNIKAR, CHANG-SHOU LIN
}

\begin{abstract}
The aim of this paper is to complete the program initiated in [50], 23 and then carried out by several authors concerning non-degeneracy and uniqueness of solutions to mean field equations. In particular, we consider mean field equations with general singular data on non-smooth domains. The argument is based on the Alexandrov-Bol inequality and on the eigenvalues analysis of linearized singular Liouville-type problems.
\end{abstract}

\section{IntRoduction}

We are concerned with the following Liouville-type problem

$$
\begin{cases}\Delta v+\rho \frac{e^{v}}{\int_{\Omega} e^{v} d x}=4 \pi \sum_{j=1}^{N} \alpha_{j} \delta_{p_{j}} & \text { in } \Omega, \\ v=0 & \text { on } \partial \Omega,\end{cases}
$$

and with its generalization in (5) below, where $\Omega \subset \mathbb{R}^{2}$ is a simply-connected, open and bounded domain, $\rho$ is a positive parameter, $\left\{p_{1}, \ldots, p_{N}\right\} \subset \Omega$ and $\alpha_{j}>-1$ for $j=1, \ldots, N$. The latter equation arises as a mean field limit of turbulent Euler flows 21,22,26, 40. Its counterpart on manifolds is related to the Electroweak and Chern-Simons self-dual vortices [49,51,54 and to the prescribed Gaussian curvature problem on surfaces 24, 25, 39, 52. Due to its relevance in mathematics and physics the literature for equation (3) is huge and we just mention [2, 3, 6, 8, 10, 15, 17, 18, 20, 27, 29, 31, 33, 35, 37, 42, 46, 48, 50,55, and the references quoted therein.

To describe the main features of the problem we first write (10) as follows. Letting $G_{p}, p \in \Omega$, be the Green function,

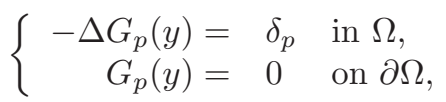

2000 Mathematics Subject Classification. 35J61, 35R01, 35A02, 35B06.

Key words and phrases. Singular Liouville-type equations, Singular Mean field equations, Nondegeneracy, Uniqueness results, Alexandrov-Bol inequality.

D.B. and A.J. are partially supported by PRIN project 2012, ERC PE1_11, "Variational and perturbative aspects in nonlinear differential problems" and by the Consolidate the Foundations project 2015 "Nonlinear Differential Problems and their Applications" (sponsored by Univ. of Rome "Tor Vergata").

D.B. is partially supported by the Mission Sustainability project 2017 "SEEA" (sponsored by Univ. of Rome "Tor Vergata") and by "Fondo per le attività base di ricerca" MIUR 2017. 
we say that $v$ is a solution of (10) if $u=v+4 \pi \sum_{j=1}^{N} \alpha_{j} G_{p_{j}}(x)$ is an $H_{0}^{1}(\Omega)$ weak solution of,

$$
\begin{cases}\Delta u+\rho \frac{V(x) e^{u}}{\int_{\Omega} V(x) e^{u} d x}=0 & \text { in } \Omega, \\ u=0 & \text { on } \partial \Omega\end{cases}
$$

where

$$
\begin{aligned}
& V(x)=\exp \left(-4 \pi \sum_{j=1}^{N} \alpha_{j} G_{p_{j}}(x)\right), \\
& V>0 \text { in } \Omega \backslash\left\{p_{1}, \ldots, p_{N}\right\}, \quad V(x) \simeq\left|x-p_{j}\right|^{2 \alpha_{j}} \text { near } p_{j} .
\end{aligned}
$$

To avoid technicalities we postpone the discussion concerning the regularity assumptions on $\Omega$ to the sequel. Problem (3) admits a variational formulation and, by a suitable adaptation of well known arguments 47, 52, the corresponding functional is seen to be coercive for $\rho<8 \pi\left(1+\min _{j}\left\{\alpha_{j}, 0\right\}\right)$. Therefore, in this range, weak solutions of (3) are obtained by direct minimization. On the other hand, the nondegeneracy and uniqueness of solutions was first proved in 50, where the author solves the regular case $(N=0)$ for $\rho<8 \pi$ and $\Omega$ smooth and simply-connected. Later, this result was improved in 23 to include the more delicate critical value $\rho=8 \pi$, and finally generalized to the case of a possibly multiply-connected domain $\Omega$ in $[13$. The argument was also refined in 12 to cover the singular case where $\rho \leq 8 \pi$ and $\alpha_{j}>0$ for all $j=1, \ldots, N, N \geq 1$. Let us point out that the latter results are sharp in the sense that uniqueness does not hold in general if $\rho>8 \pi$, see for example [6,23. More recently, in [53] the authors considered the case of one negative singularity, i.e. $\alpha_{1} \in(-1,0)$ and at least one positive singularity $\alpha_{j}>0$ for all $j=2, \ldots, N, N \geq 2$, proving non-degeneracy and uniqueness of solutions provided $\rho \leq 8 \pi\left(1+\alpha_{1}\right)$.

There is a common strategy in the above mentioned results, which is based on rearrangement type arguments and the Alexandrov-Bol inequality, whose aim is to show that the first eigenvalue of the linearized problem of Liouville-type equations (3) is strictly positive for $\rho \leq 8 \pi\left(1+\min _{j}\left\{\alpha_{j}, 0\right\}\right)$. This point is not trivial since the nonlinear term in (3) is constrained in $L^{1}(\Omega)$, whence, roughly speaking, the associated first eigenvalue "looks like" an higher order eigenvalue of a suitable unconstrained problem. As a matter of fact, for multiple negative sources (and even for a single negative source with no extra positive sources), the latter strategy is missing. Therefore, the first aim of this paper is to complete the above program and to prove non-degeneracy of solutions for (11) for general singular data on nonsmooth domains. Actually, we will develop the strategy for a much more general problem, see (5) below, in which the sum of Dirac deltas may be replaced by a general measure of bounded variation. This is somehow the more general form of the singularities which one can attach to the Dirichlet problem for (3) as it naturally arises in the analysis of Alexandrov surfaces with bounded integral curvature, see [5] and references therein.

Once we have the non degeneracy, then the uniqueness of solutions to (3), (5) will follow by the implicit function theorem and some uniform estimates for solutions to (5) with $\rho$ below the uniqueness threshold (see Theorem 1.2 below). At least to our knowledge these estimates are known only in the model case (3)-(4), as first 
derived in [20, 43] and then in [17] and [16] via blow up analysis. However, it is not easy to generalize such a refined blow-up argument to the general singular weight in (5). Also, the needed estimates were first derived in [1, but only in the analytic framework, and in [4 for weak subsolutions, but only for the model case (3)-(44). We solve this problem here by proving some uniform estimates of independent interest for weak solutions of (5), in the same spirit of [1,4].

We finally remark that the uniqueness part concerning solutions to (5) was very recently obtained in 9 by a completely different argument (see also 37] for a similar application of the latter method). From this point of view, we come up with a new proof of the uniqueness based on the non-degeneracy of (5).

In order to introduce the problem let us fix the setting and some notations. As in 23 we consider the following set of non-smooth domains.

Definition 1.1. We say that $\Omega$ is regular if its boundary is of class $C^{2}$ but for a finite number of points $\left\{Q_{1}, \ldots, Q_{N_{0}}\right\} \subset \partial \Omega$ such that the following conditions holds at each $Q_{j}$ :

(i) The inner angle $\theta_{j}$ of $\partial \Omega$ at $Q_{j}$ satisfies $0<\theta_{j} \neq \pi<2 \pi$;

(ii) At each $Q_{j}$ there is an univalent conformal map from $B_{\delta}\left(Q_{j}\right) \cap \bar{\Omega}$ to the complex plane $\mathbb{C}$ such that $\partial \Omega \cap B_{\delta}\left(Q_{j}\right)$ is mapped to a $C^{2}$ curve.

Obviously any non-degenerate polygon is regular according to this definition. Letting now $\Omega$ be regular, we will be interested in the following problem,

$$
\begin{cases}\Delta u+\rho \frac{h(x) e^{u}}{\int_{\Omega} h(x) e^{u} d x}=0 & \text { in } \Omega, \\ u=0 & \text { on } \partial \Omega .\end{cases}
$$

where $h=e^{H}$ is such that,

$$
H=\mathcal{H}_{+}-\mathcal{H}_{-},
$$

with $\mathcal{H}_{+}, \mathcal{H}_{-}$two superharmonic functions defined by

$$
\mathcal{H}_{ \pm}(x)=\mathfrak{h}_{ \pm}(x)+\int_{\Omega} G_{x}(y) d \mu_{ \pm}(y),
$$

where $\mathfrak{h}_{ \pm} \in C^{2}(\Omega) \cap C^{0}(\bar{\Omega})$ are harmonic functions in $\Omega$ and $\mu_{ \pm}$are non-negative and mutually orthogonal measures of bounded total variation compactly supported in $\Omega$.

Definition 1.2. Let $\omega \subseteq \Omega$ be a nonempty subdomain. We denote by $\widetilde{\omega}$ the interior of the closure of the union of $\omega$ with its "holes", that is, with the bounded component of the complement of $\omega$ in $\mathbb{R}^{2}$.

Definition 1.3. Let $h=e^{H}$ and let $\mu_{+}$be defined as in (6). Let $\omega \subseteq \Omega$ be a nonempty subdomain and let $\widetilde{\omega}$ be given as in Definition 1.2. We define $\alpha(\omega)=$ $\alpha(\omega, h) \geq 0$ to be

$$
\alpha(\omega)=\frac{1}{4 \pi} \mu_{+}(\widetilde{\omega})
$$

Moreover, we assume that

$$
\alpha(\Omega)<1 \text { i.e. } \mu_{+}(\Omega)<4 \pi \text {. }
$$

We remark that this is a rather natural condition, as it is somehow the minimal requirement needed to ensure that $h=e^{H} \in L^{1}(\Omega)$, whenever the measure $\mu_{+}$is 
concentrated in just a single Dirac delta, which is the model problem (3)-(4) with $N=1$.

Remark 1.1. By our assumptions on $h$, it is not difficult to see that $h$ is uniformly bounded from above and from below in a sufficiently small neighbourhood of $\partial \Omega$. As a consequence, we can argue as in Lemma 2.1 in [23] and prove that indeed $u \in C^{0}(\bar{\Omega})$.

Next, since $\mu_{+}(\Omega)<4 \pi$, then there exists at most one point $x_{0} \in \Omega$ such that $\mu_{+}\left(x_{0}\right) \geq 2 \pi$. As a consequence, by arguing as in [5], if $h e^{u} \in L^{1}(\Omega)$ where $u \in L^{1}(\Omega)$ is a solution of (5) just in the sense of distributions, then we gain $u \in W^{2, q}(\Omega)$ for some $q>1$ and in particular, for each $r>0$ small enough, there exists $s_{r}>2$ such that $u \in W^{2, s_{r}}\left(\Omega \backslash B_{r}\left(x_{0}\right)\right)$. We will refer to the latter property by saying that $u \in W^{2, s, \operatorname{loc}}\left(\Omega \backslash\left\{x_{0}\right\}\right)$ for some $s>2$. Clearly $u$ is a strong solution of (5) and similar integrability properties are deduced also on the weight $h$, see Proposition 1.4 in [5].

Our main result is the following.

Theorem 1.2. Let $\Omega$ be an open, bounded, simply-connected and regular (according to Definition 1.1) domain. Let $h$ be such that $\alpha=\alpha(\Omega, h)<1$, with $\alpha(\Omega, h)$ defined as in (7). Then, for any $\rho \leq 8 \pi(1-\alpha)$, there exists at most one weak $H_{0}^{1}(\Omega)$ solution of (5) and the first eigenvalue of the corresponding linearized problem is strictly positive.

Observe that, by choosing $h(x) \equiv V(x)$, with $V$ given in (4), and letting

$$
J=\left\{j \in\{1, \ldots, N\}: \alpha_{j} \in(-1,0)\right\},
$$

then $\alpha=\alpha(\Omega, h)>0$, as defined in (17), is given by

$$
\alpha=-\sum_{j \in J} \alpha_{j}
$$

Then, Theorem 1.2 yields the following immediate Corollary for solutions of (3).

Corollary 1.3. Let $\Omega$ be an open, bounded, simply-connected and regular (according to Definition 1.1) domain. Let $\alpha<1$ be given as in (9). Then, for any $\rho \leq 8 \pi(1-$ $\alpha$ ), there exists at most one weak $H_{0}^{1}(\Omega)$ solution of (3) and the first eigenvalue of the corresponding linearized problem is strictly positive.

As remarked above, the functional corresponding to (3) is coercive for $\rho<8 \pi(1+$ $\left.\min _{j}\left\{\alpha_{j}, 0\right\}\right)$. Therefore, we have existence and uniqueness in Corollary 1.3 if either $|J| \geq 2$ and $\rho \leq 8 \pi(1-\alpha)$ or if $|J|=1$ and $\rho<8 \pi(1-\alpha) \equiv 8 \pi\left(1+\alpha_{1}\right), \alpha_{1}<0$, or if $|J|=0$ and $\rho<8 \pi$. Now, if $|J|=N=1, p_{1}=0$ and $\Omega=B_{1}(0)$, then solutions to (3) (which are radial and well known in this particular case) exist if and only if $\rho<8 \pi(1-\alpha) \equiv 8 \pi\left(1+\alpha_{1}\right)$, showing that our existence and uniqueness result is sharp in this case. On the other side, we stress that both our result and the one in [9] yield to the same uniqueness threshold which, for $|J| \geq 2$, is lower than the subcritical existence threshold $8 \pi\left(1+\min _{j}\left\{\alpha_{j}, 0\right\}\right)$. This motivates the following interesting open problem:

Open problem. Does uniqueness of solutions for (3)-(4) hold for

$$
\rho \in\left(8 \pi(1-\alpha), 8 \pi\left(1+\min _{j}\left\{\alpha_{j}, 0\right\}\right)\right), \quad \alpha>0,
$$


with $|J| \geq 2$ ?

The strategy to prove Theorem 1.2 is inspired by the one in 23, with several non-trivial improvements needed to deal with general singular data (given by some measures as in (6) ) and non-smooth domains. To this end, the first tool we need is an Alexandrov-Bol's inequality for solutions of (5) suitable for our setting. Such an inequality was first proved in the analytical framework in [1] and more recently generalized to the weak setting in [4,5]. However what we need here is a more general statement which allows one to push the inequality, still in this weak setting, up to the (non-smooth) boundary of the domain. At least to our knowledge this is still missing and this is why we will derive it here.

Next, we perform a rearrangement argument jointly with a comparison method to gather some information about the eigenvalues of the linearized problem for (5). Here, we present some novelties.

On one side we generalize the argument in [23] (introduced to treat the regular problem $N=0$ in the more subtle case $\rho=8 \pi$ ) to the singular setting and on the other side we extend it to the sub-critical case $\rho<8 \pi(1-\alpha)$. We point out that this step is new also for the regular problem as it simplifies the original argument in [50. Special attention is also paid for the case $\rho=8 \pi(1-\alpha)$ where one has to exploit the characterization of the equality in the Alexandrov-Bol inequality recently obtained in [5] which, roughly speaking, asserts that equality can be attained only on simplyconnected subdomains $\omega \subseteq \Omega$ such that the full measure $\mu_{+}$in (7) is concentrated in just one Dirac delta. Moreover, from the equality case in some Cauchy-Schwartz inequality, we will use the boundary regularity assumptions on the domain, see Definition 1.1, in order to eventually apply the Hopf boundary lemma and get the desired conclusion. This step is done in the same spirit of [23] and the boundary regularity assumptions are crucial at this point. This is in striking contrast with [12, 53, where one can exploit the presence of positive singularities to readily conclude the argument even under weaker boundary regularity assumptions.

This paper is organized as follows. In section 2 we introduce the Alexandrov-Bol inequality and analyze the linearized Liouville-type problem. In section 3 we prove some uniform estimates for solutions to (5) and then deduce Theorem 1.2

\section{EigenVAlues ANALYSiS FOR Liouville-TyPe LiNEARIZED PROBLEMS}

In this section we first introduce the Alexandrov-Bol inequality suitable for our setting and then carry out an eigenvalues analysis for Liouville-type linearized problems which will be crucially used in the next section in the proof of Theorem 1.2 .

Definition 2.1. We say that an open set $\Omega_{0} \subset \mathbb{R}^{2}$ is simple if $\partial \Omega_{0}$ is a rectifiable Jordan curve whose interior is $\Omega_{0}$.

Clearly any regular domain according to Definition 1.1 is also simple. Next, given $\alpha \in[0,1), \lambda>0$ we set

$$
U_{\lambda, \alpha}(x)=\ln \left(\frac{\lambda(1-\alpha)}{1+\frac{\lambda^{2}}{8}|x|^{2(1-\alpha)}}\right)^{2},
$$

which satisfies

$$
\Delta U_{\lambda, \alpha}+|x|^{-2 \alpha} e^{U_{\lambda, \alpha}}=0 \text { in } \mathbb{R}^{2} \backslash\{0\} .
$$


The following version of the Alexandrov-Bol inequality was first proved in the analytical framework in [1] and more recently generalized to the weak setting in [4,5. Actually, if $\omega$ in the statement is a relatively compact subset of $\Omega_{0}$, then the result is just a particular case of Theorem 1.5 in [5].

Proposition 2.1. Let $\Omega_{0} \subset \mathbb{R}^{2}$ be a simple domain according to Definition 2.1. Let $x_{0} \in \Omega_{0}$ be fixed as in Remark 1.1 and let $w \in W^{2, s, \text { loc }}\left(\Omega_{0} \backslash\left\{x_{0}\right\}\right) \cap W^{2, q}\left(\Omega_{0}\right) \cap$ $C^{0}\left(\bar{\Omega}_{0}\right)$ for some $s>2$ and some $q>1$, satisfy

$$
\Delta w+h(x) e^{w}=0 \text { in } \Omega_{0},
$$

where $h=e^{H}$ is such that $\alpha\left(\Omega_{0}, h\right)$ (as defined in (17) satisfies $\alpha\left(\Omega_{0}, h\right)<1$. Let $\omega \subseteq \Omega_{0}$ be any open subdomain such that $\partial \omega$ is a finite union of rectifiable Jordan curves and let $\alpha(\omega)=\alpha(\omega, h)$. Then it holds,

$$
\left(\int_{\partial \omega}\left(h(x) e^{w}\right)^{\frac{1}{2}} d \sigma\right)^{2} \geq \frac{1}{2}\left(\int_{\omega} h(x) e^{w} d x\right)\left(8 \pi(1-\alpha(\omega))-\int_{\omega} h(x) e^{w} d x\right) .
$$

Moreover, the equality holds if and only if (modulo conformal transformations) $\omega=B_{\delta}(0)$ for some $\delta>0, h(x) e^{w} \equiv|x|^{-2 \alpha} e^{U_{\lambda, \alpha}}$ for some $\lambda$ where $U_{\lambda, \alpha}$ is defined in (10), $\mu_{+}=-\Delta H=4 \pi \alpha \delta_{p=0}$ in $\omega$ and $\alpha=\alpha(\omega)$. In particular, if $\omega$ is not simply-connected, then the inequality is always strict.

Proof. The proof can be worked out by a step by step adaptation of the one provided in [5] with minor changes borrowed from Theorem 4.1 in [4. Since $\omega \subseteq \Omega_{0}$ is such that $\partial \omega$ is a finite union of rectifiable Jordan curves and since $w \in C^{0}(\bar{\omega})$, then there exists $g \in C^{0}(\bar{\omega})$ such that $\Delta g=0$ in $\omega$ and $g=w$ on $\partial \omega$. Set $\eta=w-g$, $\eta \in W^{2, s, \text { loc }}\left(\omega \backslash\left\{x_{0}\right\}\right) \cap W^{2, q}(\omega) \cap C_{0}^{0}(\bar{\omega})$ for some $s>2$ and some $q>1$, which satisfies

$$
\begin{cases}\Delta \eta+h(x) e^{g} e^{\eta}=0 & \text { in } \omega, \\ \eta=0 & \text { on } \partial \omega .\end{cases}
$$

By using the strong maximum principle for weak solutions one can prove that

$$
\eta(x)>0 \forall x \in \omega \text { and } \eta(x)=0 \text { iff } x \in \partial \omega .
$$

With this at hand the strategy follows the one introduced in [5]. Since we will need some ingredients later on we sketch here the main steps and refer to [4,5, 12] for full details. We first set,

$$
\Omega(t)=\{x \in \omega: \eta(x)>t\}, \quad \Gamma(t)=\partial \Omega(t), \quad \mu(t)=\int_{\Omega(t)} h(x) e^{g} d x
$$

and observe that $\Omega(0)=\omega$. By the co-area formula one has for a.e. $t \geq 0$,

$$
\frac{d \mu(t)}{d t}=-\int_{\Gamma(t)} \frac{h(x) e^{g}}{|\nabla \eta|} d \sigma .
$$

Now, for $s \geq 0$ we define the rearrangement $\eta^{*}$ of $\eta$,

$$
\eta^{*}(s)=|\{t \geq 0: \mu(t)>s\}|,
$$

where $|E|$ is the Lebesgue measure of a Borel set $E \subset \mathbb{R}$. It is not difficult to see that $\eta^{*}$ is the inverse of $\mu$. Moreover, in [5] it is shown that $\eta^{*}$ is locally Lipschitz. 
On the other hand, by (13) one has for a.e. $s \geq 0$,

$$
\frac{d \eta^{*}(s)}{d s}=-\left(\int_{\Gamma\left(\eta^{*}(s)\right)} \frac{h(x) e^{g}}{|\nabla \eta|} d \sigma\right)^{-1} .
$$

We next define for $s \geq 0$,

$$
F(s)=\int_{\Omega\left(\eta^{*}(s)\right)} h(x) e^{\eta} e^{g} d x,
$$

and observe that $F(0)=0$ and $F(\mu(0))=M(\omega)$, where we set

$$
M(\omega)=\int_{\omega} h(x) e^{w} d x .
$$

By using the fact that $\eta^{*}$ is the inverse of $\mu$ and by (13) it is possible to check that

$$
F(s)=\int_{0}^{s} e^{\eta^{*}(\lambda)} d \lambda
$$

and hence, for a.e. $s \geq 0$,

$$
F^{\prime}(s)=e^{\eta^{*}(s)}, \quad F^{\prime \prime}(s)=\frac{d \eta^{*}(s)}{d s} e^{\eta^{*}(s)}=\frac{d \eta^{*}(s)}{d s} F^{\prime}(s) .
$$

Now, we first use the Cauchy-Schwartz inequality, then (15) and finally (12) to get for a.e. $s \geq 0$,

$$
\begin{aligned}
\left(\int_{\Gamma\left(\eta^{*}(s)\right)}\left(h(x) e^{g}\right)^{\frac{1}{2}} d \sigma\right)^{2} & \leq\left(\int_{\Gamma\left(\eta^{*}(s)\right)} \frac{h(x) e^{g}}{|\nabla \eta|} d \sigma\right)\left(\int_{\Gamma\left(\eta^{*}(s)\right)}|\nabla \eta| d \sigma\right) \\
& =\left(-\frac{d \eta^{*}(s)}{d s}\right)^{-1}\left(\int_{\Gamma\left(\eta^{*}(s)\right)}|\nabla \eta| d \sigma\right) \\
& =\left(-\frac{d \eta^{*}(s)}{d s}\right)^{-1}\left(\int_{\Omega\left(\eta^{*}(s)\right)} h(x) e^{\eta} e^{g} d x\right) \\
& =\left(-\frac{d \eta^{*}(s)}{d s}\right)^{-1} F(s) .
\end{aligned}
$$

On the other hand, the Huber inequality [38] asserts that for a.e. $s \geq 0$,

$$
\left(\int_{\Gamma\left(\eta^{*}(s)\right)}\left(h(x) e^{g}\right)^{\frac{1}{2}} d \sigma\right)^{2} \geq 4 \pi(1-\alpha(\omega)) \mu\left(\eta^{*}(s)\right)=4 \pi(1-\alpha(\omega)) s .
$$

It follows that for a.e. $s \geq 0$,

$$
4 \pi(1-\alpha(\omega)) s \leq\left(-\frac{d \eta^{*}(s)}{d s}\right)^{-1} F(s) .
$$

Letting,

$$
P(s)=4 \pi(1-\alpha(\omega))\left(s F^{\prime}(s)-F(s)\right)+\frac{1}{2} F^{2}(s),
$$

we deduce from (17) and (18) that,

$$
\frac{d}{d s} P(s) \geq 0 \text { for a.e. } s \geq 0 .
$$


Since the functions involved in the definition of $P$ are locally Lipschitz continuous, after integration we end up with,

$$
P(\mu(0))-P(0) \geq 0,
$$

which is equivalent to,

$$
8 \pi(1-\alpha(\omega))(\mu(0)-M(\omega))+M(\omega)^{2} \geq 0,
$$

where $M(\omega)$ is defined in (16). Using Huber's inequality once more we deduce that,

$$
\begin{aligned}
\left(\int_{\partial \omega}\left(h(x) e^{w}\right)^{\frac{1}{2}} d \sigma\right)^{2} & =\left(\int_{\partial \omega}\left(h(x) e^{g}\right)^{\frac{1}{2}} d \sigma\right)^{2} \\
& \geq 4 \pi(1-\alpha(\omega)) \mu(0) \\
& \geq \frac{1}{2} M(\omega)(8 \pi(1-\alpha(\omega))-M(\omega)),
\end{aligned}
$$

which is the desired inequality in (11). The characterization of the equality case can be carried out as in 5 and we skip the details.

Next we consider the eigenvalue problem for a linearized Liouville-type equation, by recalling that a nodal domain for $\phi \in C^{0}(\bar{\Omega})$ is any maximal connected component of the subdomain where $\phi$ has a definite sign. We will need a Gauss-Green formula and a Courant nodal line Theorem suitable to be applied in our weak setting. Even under our weak summability assumptions about $h$, still these results are well known, see for example [11. Therefore we omit the proof of the following Lemma which, in view of our assumptions on $h$, can be obtained by a rather standard adaptation of the one worked out in [12].

Lemma 2.2. Let $\Omega \subset \mathbb{R}^{2}$ be an open, bounded, simply-connected and piecewise $C^{2}$ domain according to Definition 1.1. Let $w-c \in H_{0}^{1}(\Omega)$ for some $c \in \mathbb{R}$ and $w$ be a weak solution of,

$$
\Delta w+h(x) e^{w}=0 \text { in } \Omega,
$$

where $h=e^{H}$ is such that $\alpha=\alpha(\Omega, h)$ (as defined in (7)) satisfies $\alpha(\Omega, h)<1$.

Suppose that for some $\hat{\nu}$, either $\phi \in H_{0}^{1}(\Omega)$ is a weak solution of,

$$
\begin{cases}-\Delta \phi-h(x) e^{w} \phi=\hat{\nu} h(x) e^{w} \phi & \text { in } \Omega, \\ \phi=0 & \text { on } \partial \Omega,\end{cases}
$$

or that exists $c_{0} \in \mathbb{R}, c_{0}<0$ such that $\phi-c_{0} \in H_{0}^{1}(\Omega)$ and $\phi$ is a weak solution of

$$
\begin{cases}-\Delta \phi-h(x) e^{w} \phi=\hat{\nu} h(x) e^{w} \phi & \text { in } \Omega, \\ \phi=c_{0} & \text { on } \partial \Omega, \\ \int_{\Omega} h(x) e^{w} \phi d x=0 . & \end{cases}
$$

Then, for a nodal domain $\omega \subseteq \Omega$ for $\phi$, it holds,

$$
-\int_{\omega} \phi \Delta \phi d x=\int_{\omega}|\nabla \phi|^{2} d x
$$

and

$$
\int_{\omega}|\nabla \phi|^{2} d x=(\hat{\nu}+1) \int_{\omega} h(x) e^{w}|\phi|^{2} d x .
$$


Moreover, let

$$
\left(\hat{\nu}_{k}, \phi_{k}^{(j)}\right) \quad k \in \mathbb{N}, \quad j=1, \ldots, l_{k}, l_{k} \in \mathbb{N}, \quad \hat{\nu}_{1}<\hat{\nu}_{2}<\ldots,
$$

be the eigenvalues and the corresponding eigenfunctions for (20). Then, $\hat{\nu}_{1}$ is simple $\left(l_{1}=1\right), \phi_{1}$ has only one nodal domain and the second eigenfunction has exactly two nodal domains. Finally, any other eigenfunction has at least two nodal domains.

With this at hand we can start the eigenvalues analysis of $\left(-\Delta-h(x) e^{w}\right)(\cdot)$. In particular, by assuming some bounds on $\int_{\Omega} h(x) e^{w} d x$ we will derive useful information on the first and second eigenvalue.

Proposition 2.3. Let $\Omega, h$ and $w$ be as in Lemma 2.2. Let $\hat{\nu}_{1}, \hat{\nu}_{2}$ be the first and second eigenvalues for (20), respectively. Then, it holds:

(i) If $\int_{\Omega} h(x) e^{w} d x<4 \pi(1-\alpha)$, then $\hat{\nu}_{1}>0$.

(ii) If $\int_{\Omega} h(x) e^{w} d x \leq 8 \pi(1-\alpha)$, then $\hat{\nu}_{2}>0$.

Proof. First of all, by Remark 1.1 we have $w \in W^{2, s, \operatorname{loc}}\left(\Omega \backslash\left\{x_{0}\right\}\right) \cap W^{2, q}(\Omega) \cap C^{0}(\bar{\Omega})$ for some $s>2, q>1$ and some $x_{0} \in \Omega$. Similar regularity properties are deduced on the eigenfunctions $\phi$ of (20). Clearly, an eigenvalue and eigenfunction $(\hat{\nu}, \phi)$ for (20) correspond to an eigenvalue and eigenfunction $(\nu=\hat{\nu}+1, \phi)$ for,

$$
\begin{cases}-\Delta \phi=\nu h(x) e^{w} \phi & \text { in } \Omega, \\ \phi=0 & \text { on } \partial \Omega .\end{cases}
$$

Proof of $(i)$. Suppose $\int_{\Omega} h(x) e^{w} d x<4 \pi(1-\alpha)$ and suppose by contradiction that there exists $\phi=\phi_{1}$, the first eigenfunction for (20), with $\hat{\nu}_{1} \leq 0$. Then, we have

$$
\begin{cases}-\Delta \phi=\nu_{1} h(x) e^{w} \phi & \text { in } \Omega, \\ \phi=0 & \text { on } \partial \Omega,\end{cases}
$$

with $\nu_{1} \leq 1$. Moreover, by Lemma 2.2 we know that $\phi$ has only one nodal domain and w.l.o.g. we assume $\phi \geq 0$ in $\Omega$. In particular, by the maximum principle for weak solutions we have $\phi>0$ in $\Omega$. Recalling (10) we set $U_{\alpha}(x):=U_{1, \alpha}(x)$, i.e.

$$
U_{\alpha}(x)=\ln \left(\frac{(1-\alpha)}{1+\frac{1}{8}|x|^{2(1-\alpha)}}\right)^{2},
$$

which satisfies,

$$
\Delta U_{\alpha}+|x|^{-2 \alpha} e^{U_{\alpha}}=0 \text { in } \mathbb{R}^{2} \backslash\{0\} .
$$

Next, let $t_{+}=\max _{\bar{\Omega}} \phi$ and for $t>0$ we define $\Omega_{t}=\{x \in \Omega: \phi>t\}$ and $R(t)>0$ such that

$$
\int_{B_{R}(t)}|x|^{-2 \alpha} e^{U_{\alpha}} d x=\int_{\Omega_{t}} h(x) e^{w} d x .
$$

Since $\phi>0$ in $\Omega$ we put $\Omega_{0}=\Omega$. Moreover, $R_{0}=\lim _{t \rightarrow 0^{+}} R(t)$ and $\lim _{t \rightarrow\left(t_{+}\right)^{-}} R(t)=$ 0 . Then $\phi^{*}: B_{R_{0}} \rightarrow \mathbb{R}$, which for $y \in B_{R_{0}},|y|=r$, is given by,

$$
\phi^{*}(r)=\sup \left\{t \in\left(0, t_{+}\right): R(t)>r\right\}
$$


is a radial, decreasing, equimeasurable rearrangement of $\phi$ with respect to the measures $h(x) e^{w} d x$ and $|x|^{-2 \alpha} e^{U_{\alpha}}$, and hence, in particular,

$$
\begin{aligned}
& B_{R(t)}=\left\{x \in \mathbb{R}^{2}: \phi^{*}(x)>t\right\}, \\
& \int_{\left\{\phi^{*}>t\right\}}|x|^{-2 \alpha} e^{U_{\alpha}} d x=\int_{\Omega_{t}} h(x) e^{w} d x \quad t \in\left[0, t_{+}\right), \\
& \int_{B_{R_{0}}}|x|^{-2 \alpha} e^{U_{\alpha}}\left|\phi^{*}\right|^{2} d x=\int_{\Omega} h(x) e^{w}|\phi|^{2} d x .
\end{aligned}
$$

Clearly, $\phi^{*}$ is a BV function. We apply the Cauchy-Schwartz inequality and the co-area formula to get that,

$$
\begin{aligned}
\int_{\{\phi=t\}}|\nabla \phi| d \sigma & \geq\left(\int_{\{\phi=t\}}\left(h(x) e^{w}\right)^{\frac{1}{2}} d \sigma\right)^{2}\left(\int_{\{\phi=t\}} \frac{h(x) e^{u}}{|\nabla \phi|} d \sigma\right)^{-1} \\
& =\left(\int_{\{\phi=t\}}\left(h(x) e^{w}\right)^{\frac{1}{2}} d \sigma\right)^{2}\left(-\frac{d}{d t} \int_{\Omega_{t}} h(x) e^{w} d x\right)^{-1},
\end{aligned}
$$

for a.e. $t$. Then, by means of the Alexandrov-Bol inequality in Proposition 2.1 we have,

$$
\begin{aligned}
& \left(\int_{\{\phi=t\}}\left(h(x) e^{w}\right)^{\frac{1}{2}} d \sigma\right)^{2}\left(-\frac{d}{d t} \int_{\Omega_{t}} h(x) e^{w} d x\right)^{-1} \\
& \geq \frac{1}{2}\left(\int_{\Omega_{t}} h(x) e^{w} d x\right)\left(8 \pi(1-\alpha)-\int_{\Omega_{t}} h(x) e^{w} d x\right)\left(-\frac{d}{d t} \int_{\Omega_{t}} h(x) e^{w} d x\right)^{-1} .
\end{aligned}
$$

Since $\phi^{*}$ is an equimeasurable rearrangement of $\phi$ with respect to the measures $h(x) e^{u} d x,|x|^{-2 \alpha} e^{U_{\alpha}} d x$, and since $|x|^{-2 \alpha} e^{U_{\alpha}}$ realizes the equality in Proposition 2.1. we get,

$$
\begin{aligned}
& \frac{1}{2}\left(\int_{\Omega_{t}} h(x) e^{w} d x\right)\left(8 \pi(1-\alpha)-\int_{\Omega_{t}} h(x) e^{w} d x\right)\left(-\frac{d}{d t} \int_{\Omega_{t}} h(x) e^{w} d x\right)^{-1} \\
& =\frac{1}{2}\left(\int_{\left\{\phi^{*}>t\right\}}|x|^{-2 \alpha} e^{U_{\alpha}} d x\right)\left(8 \pi(1-\alpha)-\int_{\left\{\phi^{*}>t\right\}}|x|^{-2 \alpha} e^{U_{\alpha}} d x\right)\left(-\frac{d}{d t} \int_{\left\{\phi^{*}>t\right\}}|x|^{-2 \alpha} e^{U_{\alpha}} d x\right)^{-1} \\
& =\left(\int_{\left\{\phi^{*}=t\right\}}\left(|x|^{-2 \alpha} e^{U_{\alpha}}\right)^{\frac{1}{2}} d \sigma\right)^{2}\left(-\frac{d}{d t} \int_{\left\{\phi^{*}>t\right\}}|x|^{-2 \alpha} e^{U_{\alpha}} d x\right)^{-1} \\
& =\int_{\left\{\phi^{*}=t\right\}}\left|\nabla \phi^{*}\right| d \sigma
\end{aligned}
$$

where in the last equality we used the co-area formula for BV functions, see 34. Therefore, we have proved that,

$$
\int_{\left\{\phi^{*}=t\right\}}\left|\nabla \phi^{*}\right| d \sigma \leq \int_{\{\phi=t\}}|\nabla \phi| d \sigma
$$

for a.e. $t$, which in turn yields

$$
\int_{B_{R_{0}}}\left|\nabla \phi^{*}\right|^{2} d x \leq \int_{\Omega}|\nabla \phi|^{2} d x
$$


With the latter estimate at hand we can use (26) and (23) (or the variational characterization of $\phi$ ) to deduce that,

$$
\begin{gathered}
\int_{B_{R_{0}}}\left|\nabla \phi^{*}\right|^{2} d x-\int_{B_{R_{0}}}|x|^{-2 \alpha} e^{U_{\alpha}}\left|\phi^{*}\right|^{2} d x \leq \int_{\Omega}|\nabla \phi|^{2} d x-\int_{\Omega} h(x) e^{w}|\phi|^{2} d x \\
=\left(\nu_{1}-1\right) \int_{\Omega} h(x) e^{w}|\phi|^{2} d x \leq 0,
\end{gathered}
$$

since $\nu_{1} \leq 1$ by assumption. Moreover, $\phi^{*}\left(R_{0}\right)=0$. Therefore, we conclude that the first eigenvalue for $\left(-\Delta-h(x) e^{w}\right)(\cdot)$ on $B_{R_{0}}$ with Dirichlet boundary conditions is non-positive. Consider now $\psi(x)=\frac{8-|x|^{2(1-\alpha)}}{8+|x|^{2(1-\alpha)}}$ which satisfies

$$
-\Delta \psi-|x|^{-2 \alpha} e^{U_{\alpha}} \psi=0 \text { in } \mathbb{R}^{2} \backslash\{0\}, \quad \psi \in H_{0}^{1}\left(B_{R}(0)\right), R=8^{\frac{1}{2(1-\alpha)}} .
$$

Since the first eigenvalue is non-positive one can deduce that $R_{0} \geq 8^{\frac{1}{2(1-\alpha)}}$. Moreover,

$$
8 \pi(1-\alpha) \frac{R_{0}^{2(1-\alpha)}}{8+R_{0}^{2(1-\alpha)}}=\int_{B_{R_{0}}}|x|^{-2 \alpha} e^{U_{\alpha}} d x=\int_{\Omega} h(x) e^{w} d x<4 \pi(1-\alpha),
$$

by assumption and hence $R_{0}<8^{\frac{1}{2(1-\alpha)}}$, yielding a contradiction.

Proof of $(i i)$. Suppose now $\int_{\Omega} h(x) e^{w} d x \leq 8 \pi(1-\alpha)$ and suppose by contradiction that there exists $\phi=\phi_{2}$, a second eigenfunction for (24) corresponding to a second eigenvalue $\nu_{2}$ with $\nu_{2}=\hat{\nu}_{2}+1 \leq 1$. From Lemma 2.2 we know that $\phi$ has exactly two nodal domains:

$$
\Omega_{+}=\{x \in \Omega: \phi(x)>0\}, \quad \Omega_{-}=\{x \in \Omega: \phi(x)<0\} .
$$

Suppose first that,

$$
\int_{\Omega_{+}} h(x) e^{w} d x<4 \pi(1-\alpha) .
$$

In this case we exploit the rearrangement argument introduced in the proof of $(i)$ and just replace $\Omega$ with $\Omega_{+}$. By using also (23) we end up with

$$
\begin{gathered}
\int_{B_{R_{0}}}\left|\nabla \phi^{*}\right|^{2} d x-\int_{B_{R_{0}}}|x|^{-2 \alpha} e^{U_{\alpha}}\left|\phi^{*}\right|^{2} d x \leq \int_{\Omega_{+}}|\nabla \phi|^{2} d x-\int_{\Omega_{+}} h(x) e^{w}|\phi|^{2} d x \\
=\left(\nu_{2}-1\right) \int_{\Omega_{+}} h(x) e^{w}|\phi|^{2} d x \leq 0,
\end{gathered}
$$

and then the same argument used in the proof of $(i)$ yields to a contradiction.

If instead $\int_{\Omega_{+}} h(x) e^{w} d x>4 \pi(1-\alpha)$ we may switch the role of $\Omega_{+}$and $\Omega_{-}$and apply again the above argument. Therefore, we are left with the case

$$
\int_{\Omega_{+}} h(x) e^{w} d x=\int_{\Omega_{-}} h(x) e^{w} d x=4 \pi(1-\alpha), \quad \int_{\Omega} h(x) e^{w} d x=8 \pi(1-\alpha) .
$$

In this case we necessarily have $R_{0}=8^{\frac{1}{2(1-\alpha)}}$ and the first eigenvalue for $(-\Delta-$ $\left.|x|^{-2 \alpha} e^{U_{\alpha}}\right)(\cdot)$ on $B_{R_{0}}$ is zero. Therefore,

$$
\int_{B_{R_{0}}}\left|\nabla \phi^{*}\right|^{2} d x-\int_{B_{R_{0}}}|x|^{-2 \alpha} e^{U_{\alpha}}\left|\phi^{*}\right|^{2} d x=0
$$


and hence, in particular, the inequality in (31) turns out to be an equality. This yields to the equality in (28) as well. On one side, the latter equality holds if and only if we have equality in the Alexandrov-Bol inequality (11) and hence, in particular, $\Omega_{+}$is simply-connected and $h(x) e^{w}$ is such that $\mu_{+}=-\Delta H=4 \pi \alpha \delta_{p}$ in $\Omega_{+}$. The same holds for $\Omega_{-}$. At this point, if we were in presence of positive singular sources, then the latter facts would force the positive singular sources to be supported on the nodal line of $\phi$ in $\Omega$ thus contradicting a result of [19] (see [12] for details) and the conclusion would follow. The general case is more delicate.

The equality in the Cauchy-Schwartz inequality (27) implies, for a.e. $t$,

$$
h(x) e^{w(x)}=c_{t}|\nabla \phi(x)|^{2},
$$

for some constant $c_{t}$ depending on $t$, for all $x$ such that $\phi(x)=t$. Since both $\Omega_{+}$and $\Omega_{-}$are simply-connected, the nodal line, which is the closure of $\{\phi(x)=0: x \in \Omega\}$, must intersect $\partial \Omega$ and moreover, we may assume without loss of generality that $\partial \Omega_{+} \cap \partial \Omega$ contains an arc of positive length. Now, since $w=0$ on $\partial \Omega$ and since $h$ is uniformly bounded from above and from below in a sufficiently small neighborhood of $\partial \Omega$, see Remark 1.1, by letting $t \rightarrow 0$ we deduce from (32) that $\frac{|\nabla \phi|}{h}$ is constant on any $C^{2}$ portion of $\partial \Omega_{+} \cap \partial \Omega$.

At this point, let $x_{0}$ be a point of the intersection of the nodal line with $\partial \Omega$. If $x_{0}$ is a smooth point of the boundary we readily have $\phi \in C^{1, \beta}, \beta \in(0,1)$ at $x_{0}$ and necessarily $\left|\nabla \phi\left(x_{0}\right)\right|=0$. Since $\frac{|\nabla \phi|}{h}$ is constant, it follows that $|\nabla \phi|=0$ on the $C^{2}$ portion of $\partial \Omega_{+} \cap \partial \Omega$ containing $x_{0}$. This is in contradiction to the Hopf boundary point lemma. If $x_{0}$ is not a smooth point of the boundary we can proceed as in Case 2 of Lemma 4.3 in 23 and exploit the properties on $\partial \Omega$ as given in Definition 1.1 to get an analogous contradiction to the Hopf lemma.

We consider now the eigenvalue problem with non-null boundary conditions (21) and follow an argument in 23. In particular, we generalize the proof in 23 to the singular case and extend it to the sub-critical regime $\rho<8 \pi(1-\alpha)$. This step is new also for the regular case and simplifies the original argument due to [50. We have the following property.

Proposition 2.4. Let $\Omega, h$ and $w$ be as in Lemma 2.2. Let $\hat{\nu}$ be an eigenvalue for (21). If $\int_{\Omega} h(x) e^{w} d x \leq 8 \pi(1-\alpha)$, then $\hat{\nu}>0$.

Proof. Suppose by contradiction that there exists an eigenfunction $\phi$ such that,

$$
\begin{cases}-\Delta \phi=\nu h(x) e^{w} \phi & \text { in } \Omega, \\ \phi=c_{0} & \text { on } \partial \Omega, \\ \int_{\Omega} h(x) e^{w} \phi d x=0, & \end{cases}
$$

for some $c_{0}<0$ and some $\nu \leq 1$. Let $\Omega_{+}$and $\Omega_{-}$be defined as in (30). We start by showing that

$$
\int_{\Omega_{+}} h(x) e^{w} d x \geq 4 \pi(1-\alpha) .
$$

First observe that clearly $\Omega_{+} \subset \subset \Omega$. The estimate in (33) will follow by showing that

$$
\int_{\omega_{+}} h(x) e^{w} d x \geq 4 \pi(1-\alpha)
$$


for any connected component $\omega_{+}$of $\Omega_{+}$. Indeed, suppose this is not the case. Then, if $\omega_{+}$is simply-connected, $\phi$ is the first eigenfunction for (20) on $\omega_{+}$and Proposition 2.3 $(i)$ implies $\hat{\nu}=\hat{\nu}_{1}>0$, a contradiction. If instead $\omega_{+}$is multiply connected it is not difficult to see that we can apply Proposition 2.3 ( $\mathrm{ii}$ ) to get $\hat{\nu}=\hat{\nu}_{2}>0$, a contradiction again. This completes the proof of (33).

Letting now

$$
\widetilde{\Omega}_{+}=\left\{x \in \Omega: \phi(x)>c_{0}\right\}, \quad \widetilde{\Omega}_{-}=\left\{x \in \Omega: \phi(x)<c_{0}\right\},
$$

we distinguish two cases.

Case 1. Suppose that $\widetilde{\Omega}_{-}=\emptyset$. In this case we perform the same rearrangement argument for $\phi$ on $\Omega$ introduced in Proposition 2.3 $(i)$. For any $t>c_{0}$ let $\phi^{*}$ be the radial, decreasing, equimeasurable rearrangement of $\phi$ with respect to the measures $h(x) e^{w} d x$ and $|x|^{-2 \alpha} e^{U_{\alpha}}$, where $U_{\alpha}$ is given in (25). In particular,

$$
\int_{B_{R_{0}}}|x|^{-2 \alpha} e^{U_{\alpha}} d x=\int_{\Omega} h(x) e^{w} d x,
$$

where $R_{0}=+\infty$ if $\int_{\Omega} h(x) e^{w} d x=8 \pi(1-\alpha)$. As in Proposition 2.3 we have,

$$
\begin{aligned}
& \int_{B_{R_{0}}}|x|^{-2 \alpha} e^{U_{\alpha}}\left|\phi^{*}\right|^{2} d x=\int_{\Omega} h(x) e^{w}|\phi|^{2} d x, \\
& \int_{B_{R_{0}}}|x|^{-2 \alpha} e^{U_{\alpha}} \phi^{*} d x=\int_{\Omega} h(x) e^{w} \phi d x=0, \\
& \int_{B_{R_{0}}}\left|\nabla \phi^{*}\right|^{2} d x \leq \int_{\Omega}|\nabla \phi|^{2} d x .
\end{aligned}
$$

Moreover,

$$
\begin{gathered}
\int_{B_{R_{0}}}\left|\nabla \phi^{*}\right|^{2} d x-\int_{B_{R_{0}}}|x|^{-2 \alpha} e^{U_{\alpha}}\left|\phi^{*}\right|^{2} d x \leq \int_{\Omega}|\nabla \phi|^{2} d x-\int_{\Omega} h(x) e^{w}|\phi|^{2} d x \\
=(\nu-1) \int_{\Omega} h(x) e^{w}|\phi|^{2} d x \leq 0 .
\end{gathered}
$$

We then define,

$$
\begin{gathered}
K^{*}=\inf \left\{\int_{B_{R_{0}}}|\nabla \psi|^{2} d x: \psi \in H_{\mathrm{rad}}\left(B_{R_{0}}\right), \int_{B_{R_{0}}}|x|^{-2 \alpha} e^{U_{\alpha}} \psi d x=0,\right. \\
\left.\int_{B_{R_{0}}}|x|^{-2 \alpha} e^{U_{\alpha}}|\psi|^{2} d x=1\right\},
\end{gathered}
$$

where $H_{\mathrm{rad}}\left(B_{R_{0}}\right)$ stands for radial functions $\psi$ with $\psi \in L^{2}\left(B_{R_{0}},|x|^{-2 \alpha} e^{U_{\alpha}} d x\right)$, $|\nabla \psi| \in L^{2}\left(B_{R_{0}}\right)$. We point out once more that $B_{R_{0}}=\mathbb{R}^{2}$ in case $\int_{\Omega} h(x) e^{w} d x=$ $8 \pi(1-\alpha)$. Observe that by construction and by the property (35) of $\phi^{*}$, we obtain $K^{*} \leq 1$ for any $\rho \leq 8 \pi(1-\alpha)$. We distinguish now between two cases.

Suppose first $\rho<8 \pi(1-\alpha)$ and $R_{0}<+\infty$. We will extend here the argument introduced in [23]. In particular, this simplifies the original argument due to [50]. We start by observing that, as in Proposition 2.3, in analogy with (29), the fact 
that $\int_{\Omega} h(x) e^{w} d x>\int_{\Omega_{+}} h(x) e^{w} d x \geq 4 \pi(1-\alpha)$, see (33), implies $R_{0}>8^{\frac{1}{2(1-\alpha)}}$. Let now $\psi^{*}$ be the minimizer of (36) which satisfies

$$
\Delta \psi^{*}+K^{*}|x|^{-2 \alpha} e^{U_{\alpha}} \psi^{*}=0 \quad \text { in } B_{R_{0}} \backslash\{0\},
$$

and

$$
\int_{B_{R_{0}}}|x|^{-2 \alpha} e^{U_{\alpha}} \psi^{*} d x=0
$$

By the latter property $\psi^{*}$ changes sign in $B_{R_{0}}$. On the other hand, we already know that $K^{*} \leq 1$ and hence $\psi^{*}$ changes sign only once otherwise we may use Proposition 2.3 ( $\mathrm{ii}$ ) to get a contradiction. Therefore, we may assume that there exists $\xi_{0} \in\left(0, R_{0}\right)$ such that,

$$
\begin{cases}\psi^{*}(r)>0 & \text { for } r \in\left[0, \xi_{0}\right) \\ \psi^{*}\left(\xi_{0}\right)=0, & \\ \psi^{*}(r)<0 & \text { for } r \in\left(\xi_{0}, R_{0}\right]\end{cases}
$$

By integrating the equation for $\psi^{*}$ and by using (37) we deduce,

$$
R_{0}\left(\psi^{*}\right)^{\prime}\left(R_{0}\right)=-K^{*} \int_{0}^{R_{0}}|s|^{-2 \alpha} e^{U_{\alpha}(s)} \psi^{*}(s) s d s=0 .
$$

Therefore, so far we can assert that,

$$
\psi^{*}\left(R_{0}\right)<0, \quad\left(\psi^{*}\right)^{\prime}\left(R_{0}\right)=0 .
$$

Consider now $\psi(x)=\frac{8-|x|^{2(1-\alpha)}}{8+|x|^{2(1-\alpha)}}$ which satisfies

$$
\begin{cases}\Delta \psi+|x|^{-2 \alpha} e^{U_{\alpha}} \psi=0 & \text { in } \mathbb{R}^{2} \backslash\{0\} \\ \psi(x)=0 & \text { for }|x|=8^{\frac{1}{2(1-\alpha)}} .\end{cases}
$$

In particular, since $R_{0}>8^{\frac{1}{2(1-\alpha)}}$ we have,

$$
\psi\left(R_{0}\right)<0, \quad \psi^{\prime}\left(R_{0}\right)<0 .
$$

We aim to show that $\xi_{0}=8^{\frac{1}{2(1-\alpha)}}$. Similarly as before, by using the equations for $\psi$ and $\psi^{*}$ it is not difficult to check that,

$$
r\left(\frac{\psi^{*}}{\psi}\right)^{\prime}(r) \psi^{2}(r)=\left(1-K^{*}\right) \int_{0}^{r}|s|^{-2 \alpha} e^{U_{\alpha}(s)} \psi^{*}(s) \psi(s) s d s .
$$

Suppose by contradiction $\xi_{0}<8^{\frac{1}{2(1-\alpha)}}$. Then, since $K^{*} \leq 1$, the right-hand side in (40) is non-negative for $r \leq \xi_{0}$ and hence $\frac{\psi^{*}(r)}{\psi(r)}$ is non-decreasing for $r \leq \xi_{0}$. It follows that,

$$
0<\frac{\psi^{*}(0)}{\psi(0)} \leq \frac{\psi^{*}\left(\xi_{0}\right)}{\psi\left(\xi_{0}\right)}=0,
$$

which is a contradiction. Now, analogous computations as before yield,

$R_{0}\left(\frac{\psi^{*}}{\psi}\right)^{\prime}\left(R_{0}\right) \psi^{2}\left(R_{0}\right)-r\left(\frac{\psi^{*}}{\psi}\right)^{\prime}(r) \psi^{2}(r)=\left(1-K^{*}\right) \int_{r}^{R_{0}}|s|^{-2 \alpha} e^{U_{\alpha}(s)} \psi^{*}(s) \psi(s) s d s$.

Observe that by (38) and (39) we have,

$$
\left(\psi^{*}\right)^{\prime}\left(R_{0}\right) \psi\left(R_{0}\right)-\psi^{\prime}\left(R_{0}\right) \psi^{*}\left(R_{0}\right)<0 .
$$


It follows that,

$$
-r\left(\frac{\psi^{*}}{\psi}\right)^{\prime}(r) \psi^{2}(r)=\left(1-K^{*}\right) \int_{r}^{R_{0}}|s|^{-2 \alpha} e^{U_{\alpha}(s)} \psi^{*}(s) \psi(s) s d s+C_{0},
$$

for some $C_{0}>0$. Suppose now by contradiction $\xi_{0}>8^{\frac{1}{2(1-\alpha)}}$. Then, the righ-hand side in (42) is positive for $r \geq \xi_{0}$ and hence $\frac{\psi^{*}(r)}{\psi(r)}$ is decreasing for $r \geq \xi_{0}$. Therefore, recalling (38) and (39),

$$
0=\frac{\psi^{*}\left(\xi_{0}\right)}{\psi\left(\xi_{0}\right)}>\frac{\psi^{*}\left(R_{0}\right)}{\psi\left(R_{0}\right)}>0
$$

which is a contradiction again. We conclude that necessarily $\xi_{0}=8^{\frac{1}{2(1-\alpha)}}$ and, in particular,

$$
\psi^{*}(r) \psi(r)>0 \quad \text { for all } r \neq 8^{\frac{1}{2(1-\alpha)}} .
$$

Finally, by using once more (42) with $r=0$ we have,

$$
\left(1-K^{*}\right) \int_{0}^{R_{0}}|s|^{-2 \alpha} e^{U_{\alpha}(s)} \psi^{*}(s) \psi(s) s d s=-C_{0}<0 .
$$

Since (43) holds true, then the latter inequality implies $K^{*}>1$ which is a contradiction. This concludes the proof of Case 1 for $\rho<8 \pi(1-\alpha)$.

Consider now the case $\rho=8 \pi(1-\alpha)$ and $B_{R_{0}}=\mathbb{R}^{2}$. The same argument adopted above in this situation shows that the quantity in (41) vanishes as $R_{0} \rightarrow+\infty$. Then we have $C_{0}=0$ in (42) and (44) which in turn imply that $K^{*}=1$. We skip the details to avoid repetitions and refer to 23 for more details concerning this point. Therefore, the inequality in (35) turns out to be an equality. This yields to the equality in (28) as well and, in particular, to the equality in the Alexandrov-Bol inequality (11). In particular,

$$
\left(\int_{\{\phi=t\}}\left(h(x) e^{w}\right)^{\frac{1}{2}} d \sigma\right)^{2}=\frac{1}{2}\left(\int_{\Omega_{t}} h(x) e^{w} d x\right)\left(8 \pi(1-\alpha)-\int_{\Omega_{t}} h(x) e^{w} d x\right),
$$

for a.e. $t>c_{0}$. However, the left-hand side of the latter equality is uniformly strictly positive, while the right-hand side tends to zero for $t \rightarrow c_{0}^{+}$. This yields to a contradiction in the case $\rho=8 \pi(1-\alpha)$ as well.

Case 2. Suppose now that $\widetilde{\Omega}_{-} \neq \emptyset$. In this case we start by considering the rearrangement $\phi^{*}$ of $\phi$ in $\widetilde{\Omega}_{+}$. In particular,

$$
\int_{B_{R_{0}^{+}}}|x|^{-2 \alpha} e^{U_{\alpha}} d x=\int_{\widetilde{\Omega}_{+}} h(x) e^{w} d x
$$

for some $R_{0}^{+}$, while $R_{0}$ is given by (34) where we recall that $R_{0}=+\infty$ if $\int_{\Omega} h(x) e^{w} d x=$ $8 \pi(1-\alpha)$ while $R_{0}<+\infty$ if $\int_{\Omega} h(x) e^{w} d x<8 \pi(1-\alpha)$. On the other hand, in $\widetilde{\Omega}_{-}$ we will consider the annular, radial, decreasing, equimeasurable rearrangement $\phi^{* *}$ of $\phi$. To this end, for any $t \in\left(t_{-}, c_{0}\right), t_{-}=\min _{\Omega} \phi$, we let $R^{-}(t)$ be such that,

$$
\int_{B_{R_{0}} \backslash B_{R^{-}(t)}}|x|^{-2 \alpha} e^{U_{\alpha}} d x=\int_{\{\phi<t\}} h(x) e^{w} d x,
$$

Then, $\phi^{* *}: B_{R_{0}} \backslash \bar{B}_{R_{0}^{+}} \rightarrow \mathbb{R}$, for $y \in B_{R_{0}} \backslash \bar{B}_{R_{0}^{+}},|y|=r$, is given by,

$$
\phi^{* *}(r)=\inf \left\{t \in\left(t_{-}, c_{0}\right): R^{-}(t)<r\right\} .
$$


We have,

$$
\begin{aligned}
& \int_{B_{R_{0}} \backslash \bar{B}_{R_{0}^{+}}}|x|^{-2 \alpha} e^{U_{\alpha}} d x=\int_{\widetilde{\Omega}_{-}} h(x) e^{w} d x, \\
& \int_{B_{R_{0}} \backslash \bar{B}_{R_{0}^{+}}}|x|^{-2 \alpha} e^{U_{\alpha}}\left|\phi^{* *}\right|^{2} d x=\int_{\widetilde{\Omega}_{-}} h(x) e^{w}|\phi|^{2} d x, \\
& \int_{B_{R_{0}} \backslash \bar{B}_{R_{0}^{+}}}|x|^{-2 \alpha} e^{U_{\alpha}} \phi^{* *} d x=\int_{\widetilde{\Omega}_{-}} h(x) e^{w} \phi d x, \\
& \int_{B_{R_{0}} \backslash \bar{B}_{R_{0}^{+}}}\left|\nabla \phi^{* *}\right|^{2} d x \leq \int_{\Omega}|\nabla \phi|^{2} d x-\int_{\widetilde{\Omega}_{+}}|\nabla \phi|^{2} d x .
\end{aligned}
$$

Finally, we let $\phi_{*}: B_{R_{0}} \rightarrow \mathbb{R}$ be the following radial function,

$$
\phi_{*}(r)= \begin{cases}\phi^{*}(r), & r \in\left[0, R_{0}^{+}\right], \\ \phi^{* *}(r), & r \in\left(R_{0}^{+}, R_{0}\right) .\end{cases}
$$

By using the properties of $\phi^{*}, \phi^{* *}$ and of $\phi$ (see also (23) ) we obtain,

$$
\begin{aligned}
\int_{B_{R_{0}}}\left|\nabla \phi_{*}\right|^{2} d x & =\int_{B_{R_{0}^{+}}}\left|\nabla \phi^{*}\right|^{2} d x+\int_{B_{R_{0}} \backslash \bar{B}_{R_{0}^{+}}}\left|\nabla \phi^{* *}\right|^{2} d x \\
& \leq \int_{\widetilde{\Omega}_{+}}|\nabla \phi|^{2} d x+\int_{\Omega}|\nabla \phi|^{2} d x-\int_{\widetilde{\Omega}_{+}}|\nabla \phi|^{2} d x \\
& =\int_{\Omega}|\nabla \phi|^{2} d x=\int_{\Omega} h(x) e^{w}|\phi|^{2} d x \\
& =\int_{B_{R_{0}^{+}}}|x|^{-2 \alpha} e^{U_{\alpha}}\left|\phi^{*}\right|^{2} d x+\int_{B_{R_{0}} \backslash \bar{B}_{R_{0}^{+}}}|x|^{-2 \alpha} e^{U_{\alpha}}\left|\phi^{* *}\right|^{2} d x \\
& =\int_{B_{R_{0}}}|x|^{-2 \alpha} e^{U_{\alpha}}\left|\phi_{*}\right|^{2} d x
\end{aligned}
$$

Thus, we conclude that,

$$
\int_{B_{R_{0}}}\left|\nabla \phi_{*}\right|^{2} d x-\int_{B_{R_{0}}}|x|^{-2 \alpha} e^{U_{\alpha}}\left|\phi_{*}\right|^{2} d x \leq 0 .
$$

Moreover, we have,

$$
\int_{B_{R_{0}}}|x|^{-2 \alpha} e^{U_{\alpha}} \phi_{*} d x=0 .
$$

At this point observe that, defining $K^{*}$ as in (36), by construction and by the property (45) of $\phi_{*}$, we obtain once more $K^{*} \leq 1$ for any $\rho \leq 8 \pi(1-\alpha)$.

We distinguish now between two cases. Suppose first that $\rho<8 \pi(1-\alpha)$. In this situation we follow step by step the argument in Case 1 (starting from (37)) to conclude that $K^{*}>1$, which is a contradiction. Suppose now that $\rho=8 \pi(1-\alpha)$ and argue once more as in Case 1 to conclude that $K^{*}=1$. Therefore, the inequality in (45) turns out to be an equality. This yields to the equality in (28) as well and, in particular, to the equality in the Alexandrov-Bol inequality (11). We conclude that necessarily $\Omega_{t}$ and $\{\phi<t\}$ are simply-connected for a.e. $t>c_{0}$. But clearly $\{\phi<t\}$ is not simply-connected for $t<c_{0}$. This yields a contradiction and the 
proof is completed.

\section{The Proof of Theorem 1.2}

In this section we start by proving some new uniform estimates for solutions to (51) and then finally deduce the main result of Theorem 1.2 by making use of the spectral estimates introduced in the previous section.

The following estimates are the counterpart of the well know results obtained in 17, 20,43 and [16 for the model case (3)-(44) in the subcritical region. However it seems not easy to adapt those results to the general case in (5). Thus we will adopt a different method based on rearrangement arguments in the same spirit of [1,4].

Proposition 3.1. Let $\Omega_{0} \subset \mathbb{R}^{2}$ be a simple domain according to Definition 2.1. Let $x_{0} \in \Omega_{0}$ be fixed as in Remark 1.1 and let $w \in W^{2, s, \text { loc }}\left(\Omega_{0} \backslash\left\{x_{0}\right\}\right) \cap W^{2, q}\left(\Omega_{0}\right) \cap$ $C^{0}\left(\bar{\Omega}_{0}\right)$ for some $s>2$ and some $q>1$, satisfy

$$
\Delta w+h(x) e^{w}=0 \text { in } \Omega_{0},
$$

where $h=e^{H}$ is such that $\alpha\left(\Omega_{0}, h\right)$ (as defined in (7)) satisfies $\alpha\left(\Omega_{0}, h\right)<1$. Let $\omega \subseteq \Omega_{0}$ be any open subdomain such that $\partial \omega$ is a finite union of rectifiable Jordan curves and let $\alpha(\omega)=\alpha(\omega, h)$. Suppose

$$
M(\omega)=\int_{\omega} h(x) e^{w} d x<8 \pi(1-\alpha(\omega)) .
$$

Then,

$$
\max _{\bar{\omega}} e^{w} \leq\left(1-\frac{M(\omega)}{8 \pi(1-\alpha(\omega))}\right)^{-2} \max _{\partial \omega} e^{w} .
$$

In particular, for any $\varepsilon \in\left(0,8 \pi\left(1-\alpha\left(\Omega_{0}\right)\right)\right)$, there exists $C_{\varepsilon}>0$ such that

$$
\left\|u_{\rho}\right\|_{L^{\infty}\left(\Omega_{0}\right)} \leq \rho C_{\varepsilon},
$$

for any $\rho \in\left(0,8 \pi\left(1-\alpha\left(\Omega_{0}\right)\right)-\varepsilon\right)$ and for any solution $u_{\rho} \in W^{2, s, \text { loc }}\left(\Omega_{0} \backslash\left\{x_{0}\right\}\right) \cap$ $W^{2, q}\left(\Omega_{0}\right) \cap C^{0}\left(\bar{\Omega}_{0}\right)$ of (5) with $\Omega=\Omega_{0}$.

Proof. Let $g$ and $\eta$ be the functions defined in the proof of Proposition 2.1 with $\eta=w-g$ and $\eta=0$ on $\partial \omega$, i.e. $g=w$ on $\partial \omega$. Let

$$
\Omega(t)=\{x \in \omega: \eta(x)>t\}, \quad \mu(t)=\int_{\Omega(t)} h(x) e^{g} d x,
$$

and let $\eta^{*}$ be the rearrangement of $\eta$ as given in (14). Finally, let $F(s)$ and $P(s)$ be defined as in the proof of Proposition 2.1 which we recall here for reader's convenience,

$$
\begin{gathered}
F(s)=\int_{\Omega\left(\eta^{*}(s)\right)} h(x) e^{\eta} e^{g} d x \\
P(s)=4 \pi(1-\alpha(\omega))\left(s F^{\prime}(s)-F(s)\right)+\frac{1}{2} F^{2}(s) .
\end{gathered}
$$

By (19) we have, for a.a. $s \in(0, \mu(0))$,

$$
P(s) \geq P(0)=0 .
$$


Let us introduce now, for a.a. $s \in(0, \mu(0))$,

$$
J(s)=s\left(\frac{1}{F(s)}-\frac{1}{8 \pi(1-\alpha(\omega))}\right),
$$

and observe that $J(s)>0$ since by assumption, $F(s) \leq F(\mu(0))=M(\omega)<$ $8 \pi(1-\alpha(\omega))$. Moreover,

$$
J^{\prime}(s)=-\frac{P(s)}{4 \pi(1-\alpha(\omega)) F^{2}(s)} \leq 0
$$

by (48). Thus, $J$ is non-increasing and in particular, for a.a. $s \in(0, \mu(0))$ we have,

$$
J(s) \leq \lim _{s \rightarrow 0^{+}} J(s)
$$

which, by the l'Hopital theorem reads,

$$
\lim _{s \rightarrow 0^{+}} J(s)=\lim _{s \rightarrow 0^{+}} \frac{s}{F(s)}=\lim _{s \rightarrow 0^{+}} \frac{1}{F^{\prime}(s)}=\frac{1}{F^{\prime}(0)} .
$$

Recalling (17) we have $F^{\prime}(0)=\max _{\bar{\omega}} e^{\eta}$ and hence, for any a.a. $s \in(0, \mu(0))$ we obtain,

$$
\max _{\bar{\omega}} e^{\eta} \leq \frac{1}{J(s)}
$$

We now estimate $J(s)$ by using (48) once more which is equivalent to, for a.a. $s \in(0, \mu(0))$,

$$
s F^{\prime}(s) \geq F^{2}(s)\left(\frac{1}{F(s)}-\frac{1}{8 \pi(1-\alpha(\omega))}\right) .
$$

Recalling that $J(s)>0$ and $F^{\prime}(s)>0$ we exploit the latter estimate to deduce, for a.a. $s \in(0, \mu(0))$,

$$
\begin{aligned}
J(s) & =\frac{s F^{\prime}(s)}{F^{\prime}(s)}\left(\frac{1}{F(s)}-\frac{1}{8 \pi(1-\alpha(\omega))}\right) \\
& \geq \frac{F(s)}{F^{\prime}(s)}\left(\frac{1}{F(s)}-\frac{1}{8 \pi(1-\alpha(\omega))}\right)^{2} \\
& =\frac{1}{F^{\prime}(s)}\left(1-\frac{F(s)}{8 \pi(1-\alpha(\omega))}\right)^{2} .
\end{aligned}
$$

Going back to (49) we obtain, for a.a. $s \in(0, \mu(0))$,

$$
\max _{\bar{\omega}} e^{\eta} \leq \frac{1}{F^{\prime}(s)}\left(1-\frac{F(s)}{8 \pi(1-\alpha(\omega))}\right)^{-2} .
$$

Finally, letting $s \rightarrow \mu(0)^{-}$we eventually get,

$$
\max _{\bar{\omega}} e^{\eta} \leq\left(1-\frac{M(\omega)}{8 \pi(1-\alpha(\omega))}\right)^{-2} .
$$


On the other hand, recall that $\eta=w-g$ with $g$ harmonic and $g=w$ on $\partial \omega$. Thus, by making use also of the weak maximum principle we obtain,

$$
\begin{aligned}
\max _{\bar{\omega}} e^{w} & =\max _{\bar{\omega}} e^{\eta+g} \leq\left(1-\frac{M(\omega)}{8 \pi(1-\alpha(\omega))}\right)^{-2} \max _{\bar{\omega}} e^{g} \\
& =\left(1-\frac{M(\omega)}{8 \pi(1-\alpha(\omega))}\right)^{-2} \max _{\partial \omega} e^{g} \\
& =\left(1-\frac{M(\omega)}{8 \pi(1-\alpha(\omega))}\right)^{-2} \max _{\partial \omega} e^{w},
\end{aligned}
$$

which is the desired estimate in (46).

We can now prove the main theorem.

Proof of Theorem 1.2. We first aim to show that the linearized equation for (5) has strictly positive first eigenvalue for any $\rho \leq 8 \pi(1-\alpha)$, where $\alpha=\alpha(\Omega, h)<1$ is given in (7). Indeed, suppose by contradiction that there exist a solution $u$ for (5) with $\rho \leq 8 \pi(1-\alpha)$ and a non-trivial eigenfunction $\tilde{\phi} \in H_{0}^{1}(\Omega)$, that is,

$$
\begin{cases}-\Delta \tilde{\phi}-\rho \frac{h(x) e^{u}}{\int_{\Omega} h(x) e^{u} d x}\left(\tilde{\phi}-\frac{\int_{\Omega} h(x) e^{u} \tilde{\phi} d x}{\int_{\Omega} h(x) e^{u} d x}\right)=0 & \text { in } \Omega, \\ \tilde{\phi}=0 & \text { on } \partial \Omega .\end{cases}
$$

Letting

$$
w=u+\log \rho-\log \left(\int_{\Omega} h(x) e^{u} d x\right), \quad \phi=\tilde{\phi}-\frac{\int_{\Omega} h(x) e^{u} \tilde{\phi} d x}{\int_{\Omega} h(x) e^{u} d x},
$$

we have,

$$
\begin{cases}-\Delta \phi-h(x) e^{w} \phi=0 & \text { in } \Omega, \\ \phi=c_{0} & \text { on } \partial \Omega, \\ \int_{\Omega} h(x) e^{w} \phi d x=0, & \end{cases}
$$

for some $c_{0} \in \mathbb{R}$. Without loss of generality we may assume $c_{0} \leq 0$. The goal is to show that $\phi \equiv c_{0}$ which in turn implies $\tilde{\phi} \equiv 0$ yielding a contradiction.

Observe that $\int_{\Omega} h(x) e^{w} \phi d x=0$. Therefore, if $c_{0}=0, \phi$ must change sign unless $\phi \equiv 0$. Then, by Lemma 2.2 $\phi$ is an eigenfunction for (20) corresponding to an eigenvalue $\hat{\nu}_{k}$ for some $k \geq 2$. On the other hand, Proposition 2.3 (ii) implies $\hat{\nu}_{2}>0$. Then, we necessarily have $\phi \equiv 0$ as claimed. We are left with the case $c_{0}<0$. But in this case we may apply Proposition 2.4 which asserts that $\hat{\nu}>0$ which is a contradiction.

Therefore, we conclude that the linearized operator $L_{\rho}$ for (5) has strictly positive first eigenvalue for any $\rho \leq 8 \pi(1-\alpha)$. Let now $S_{\rho}$ be the branch of solutions for (5) bifurcating from $(u, \rho)=(0,0)$. By standard bifurcation theory [30], we deduce that $S_{\rho}$ is a simple branch near $\rho=0$. In particular, for any $\rho>0$ small enough, there exists a unique solution for (5). Moreover, since $L_{\rho}$ has strictly positive first eigenvalue we can apply the implicit function theorem to extend uniquely $S_{\rho}$ for any $\rho<8 \pi(1-\alpha)$. Suppose by contradiction there exists a second (nonbending) branch of solutions for $\rho<8 \pi(1-\alpha)$. Then, the estimates in (47) implies that the latter branch intersects $S_{\rho}$ in $(u, \rho)=(0,0)$, which can not happen. We 
conclude uniqueness of solutions for (5) holds for $\rho<8 \pi(1-\alpha)$. Finally, assume by contradiction that there exist more than on solution for $\rho=8 \pi(1-\alpha)$. Since we can apply the implicit function theorem around each one of these solutions, then we readily obtain a contradiction to the uniqueness for $\rho<8 \pi(1-\alpha)$.

\section{REFERENCES}

[1] C. Bandle, On a differential Inequality and its applications to Geometry. Math. Zeit. 147 (1976), 253-261.

[2] D. Bartolucci, On the best pinching constant of conformal metrics on $\mathbb{S}^{2}$ with one and two conical singularities. J. Geom. Analysis 23 (2013), 855-877.

[3] D. Bartolucci, Global bifurcation analysis of mean field equations and the Onsager microcanonical description of two-dimensional turbulence. Preprint (2016), Arxiv https://arxiv.org/abs/1609.04139.

[4] D. Bartolucci, D. Castorina, Self gravitating cosmic strings and the Alexandrov's inequality for Liouville-type equations. Commun. Contemp. Math. 18 (2016), no. 4, 1550068, 26 pp.

[5] D. Bartolucci, D. Castorina, On a singular Liouville-type equation and the Alexandrov isoperimetric inequality. To appear on Ann. Scuola Norm. Sup. Pisa Cl. Sci.

[6] D. Bartolucci, F. De Marchis, Supercritical Mean Field Equations on convex domains and the Onsager's statistical description of two-dimensional turbulence. Arch. Ration. Mech. Anal. 217 (2015), no. 2, 525-570.

[7] D. Bartolucci, F. De Marchis, A. Malchiodi, Supercritical conformal metrics on surfaces with conical singularities. Int. Math. Res. Not. 24 (2011), 5625-5643.

[8] D. Bartolucci, C.C. Chen, C.S. Lin, G. Tarantello, Profile of Blow Up Solutions To Mean Field Equations with Singular Data. Comm. in P.D.E. 29 (2004), no. 7-8, 1241-1265.

[9] D. Bartolucci, C. Gui, A. Jevnikar, A. Moradifam, A singular Sphere Covering Inequality: uniqueness and symmetry of solutions to singular Liouville-type equations. Preprint (2018), Arxiv https://arxiv.org/abs/1802.09475.

[10] D. Bartolucci, A. Jevnikar, Y. Lee, W. Yang, Uniqueness of bubbling solutions of mean field equations. Preprint (2017), Arxiv https://arxiv.org/abs/1704.02354, to appear on Jour. Math. Pure App.

[11] D. Bartolucci, A. Jevnikar, Y. Lee, W. Yang, Non-degeneracy, Mean Field Equations and the Onsager theory of $2 D$ turbulence. To appear on Arch. Rat. Mech. Anal., https://doi.org/10.1007/s00205-018-1248-y.

[12] D. Bartolucci, C.S. Lin, Uniqueness Results for Mean Field Equations with Singular Data. Comm. in P.D.E. 34 (2009), no. 7, 676-702.

[13] D. Bartolucci, C.S. Lin, Existence and uniqueness for Mean Field Equations on multiply connected domains at the critical parameter. Math. Ann. 359 (2014), 1-44.

[14] D. Bartolucci, C.S. Lin, G. Tarantello, Uniqueness and symmetry results for solutions of a mean field equation on $\mathbb{S}^{2}$ via a new bubbling phenomenon. Comm. Pure Appl. Math. 64 (2011), no. 12, 1677-1730.

[15] D. Bartolucci, A. Malchiodi, An improved geometric inequality via vanishing moments, with applications to singular Liouville equations. Comm. Math. Phys. 322 (2013), 415-452.

[16] D. Bartolucci, E. Montefusco, Blow up analysis, existence and qualitative properties of solutions for the two dimensional Emden-Fowler equation with singular potential, $\mathrm{M}^{2}$.A.S. 30(18) (2007), 2309-2327.

[17] D. Bartolucci, G. Tarantello, Liouville type equations with singular data and their applications to periodic multivortices for the electroweak theory. Comm. Math. Phys. 229 (2002), 3-47.

[18] D. Bartolucci, G. Tarantello, Asymptotic blow-up analysis for singular Liouville type equations with applications. J. Diff. Eq. 262 (2017), no. 7, 3887-3931.

[19] L. Bers, (1956). Remark on an application of pseudoanalytic functions. Am. Jour. of Math. 785 (1956), 486-496.

[20] H. Brezis, F. Merle, Uniform estimates and blow-up behaviour for solutions of $-\Delta u=V(x) e^{u}$ in two dimensions. Comm. in P.D.E. 16 (1991), no. 8-9, 1223-1253. 
[21] E. Caglioti, P.L. Lions, C. Marchioro, M. Pulvirenti, A special class of stationary flows for two dimensional Euler equations: a statistical mechanics description. Comm. Math. Phys. 143 (1992), 201-525.

[22] E. Caglioti, P.L. Lions, C. Marchioro, M. Pulvirenti, A special class of stationary flows for two dimensional Euler equations: a statistical mechanics description. II. Comm. Math. Phys. 174 (1995), 229-260.

[23] S.Y.A. Chang, C.C. Chen, C.S. Lin, Extremal functions for a mean field equation in two dimension, in: "Lecture on Partial Differential Equations", New Stud. Adv. Math. 2 Int. Press, Somerville, MA, 2003, 61-93.

[24] S.Y. A. Chang and Paul C. Yang, Prescribing Gaussian curvature on $S^{2}$. Acta Math. (1987), 159(3-4), 215259.

[25] S.Y. A. Chang and Paul C. Yang, Conformal deformation of metrics on $S^{2}$. J. Differential Geom. (1988), 27(2):259296.

[26] S. Chanillo, M. Kiessling, Rotational symmetry of solutions of some nonlinear problems in statistical mechanics and in geometry. Comm. Math. Phys. 160 (1994), 217-238.

[27] C.C. Chen, C.S. Lin, Topological degree for a mean field equation on Riemann surfaces. Comm. Pure Appl. Math. 56 (2003), 1667-1727.

[28] C.C. Chen , C.S. Lin, Mean field equations of Liouville type with singular data: sharper estimates, Discr. Cont. Dyn. Syt. 28(3) (2010), 1237-1272.

[29] C.C. Chen, C.S. Lin, Mean field equation of Liouville type with singular data: topological degree. Comm. Pure Appl. Math. 68 (2015), no. 6, 887-947.

[30] M.G. Crandall, P.H. Rabinowitz, (1975). Some continuation and variational methods for positive solutions of nonlinear elliptic eigenvalue problems. Arch. Rat. Mech. An. 58 (1975), 207-218.

[31] F. De Marchis, Generic multiplicity for a scalar field equation on compact surfaces. J. Funct. An. 259 (2010), 2165-2192.

[32] W. Ding, J. Jost, J. Li, G. Wang, Existence results for mean field equations. Ann. Inst. H. Poincaré Anal. Non Linéaire 16 (1999), 653-666.

[33] Z. Djadli, Existence result for the mean field problem on Riemann surfaces of all genuses. Comm. Contemp. Math. 10 (2008), no. 2, 205-220.

[34] Fleming, R. Rishel, An integral formula for total gradient variation, Arch. Math. 11 (1960), $218-222$.

[35] C. Gui, A. Moradifam, The Sphere Covering Inequality and its applications. To appear in Invent. Math.

[36] C. Gui, A. Moradifam, Symmetry of solutions of a mean field equation on flat tori. To appear in Int. Math. Res. Not.

[37] C. Gui, A. Moradifam, Uniqueness of solutions of mean field equations in $\mathbb{R}^{2}$. To appear in Proc. Amer. Math. Soc.

[38] A. Huber, Zur Isoperimetrischen Ungleichung Auf Gekrümmten Flächen, Acta. Math. 97 (1957), 95-101.

[39] J.L. Kazdan, F.W. Warner, Curvature functions for compact 2-manifolds. Ann. Math. 99 (1974), 14-74

[40] M.H.K. Kiessling, Statistical mechanics of classical particles with logaritmic interaction. Comm. Pure Appl. Math. 46 (1993), 27-56

[41] G. Kokarev, On multiplicity bounds for Schrdinger eigenvalues on Riemannian surfaces. Analysis and PDE, 7 (2014), no. 6, 1397-1420.

[42] Y.Y. Li, Harnack type inequality: the method of moving planes, Comm. Math. Phys. 200 (1999), 421-444.

[43] Y.Y. Li, I. Shafrir, Blow-up analysis for Solutions of $-\Delta u=V(x) e^{u}$ in dimension two. Ind. Univ. Math. J. 43 (1994), no. 4, 1255-1270.

[44] C.S. Lin, Uniqueness of solutions to the mean field equations for the spherical Onsager vortex. Arch. Ration. Mech. Anal. 153 (2000), no. 2, 153-176.

[45] C.S. Lin, C.L. Wang, Elliptic functions, Green functions and the mean field equations on tori. Ann. of Math. 172 (2010), no. 2, 911-954.

[46] A. Malchiodi, Topological methods for an elliptic equation with exponential nonlinearities. Discr. Cont. Dyn. Syst. 21 (2008), 277-294.

[47] J. Moser, A sharp form of an inequality by N.Trudinger, Indiana Univ. Math. J. 20 (1971), 1077-1091. 
[48] J. Prajapat, G. Tarantello, On a class of elliptic problems in $\mathbb{R}^{2}$ : Symmetry and uniqueness results. Proc. Roy. Soc. Edinburgh Sect. A. 131 (2001), 967-985.

[49] J. Spruck, Y. Yang, On Multivortices in the Electroweak Theory I:Existence of Periodic Solutions. Comm. Math. Phys. 144 (1992), 1-16.

[50] T. Suzuki, Global analysis for a two-dimensional elliptic eiqenvalue problem with the exponential nonlinearly. Ann. Inst. H. Poincaré Anal. Non Linéaire 9 (1992), no. 4, 367-398.

[51] G. Tarantello, Multiple condensate solutions for the Chern-Simons-Higgs theory. J. Math. Phys. 37 (1996), 3769-3796.

[52] M. Troyanov, Prescribing curvature on compact surfaces with conical singularities. Trans. Amer. Math. Soc. 324 (1991), 793-821.

[53] J. Wei, L. Zhang, Non-degeneracy of Gauss curvature equation with negative conic singularity. Preprint (2017), Arxiv https://arxiv.org/abs/1706.10264, to appear on Pacific J. Math.

[54] Y. Yang, "Solitons in Field Theory and Nonlinear Analysis". Springer Monographs in Mathematics, Springer, New York, 2001.

[55] L. Zhang, Asymptotic behavior of blowup solutions for elliptic equations with exponential nonlinearity and singular data. Commun. Contemp. Math. 11 (2009), 395-411.

Daniele Bartolucci, Department of Mathematics, University of Rome "Tor Vergata", Via Della Ricerca Scientifica 1, 00133 Roma, Italy.

E-mail address: bartoluc@mat.uniroma2.it

Aleks Jevnikar, Department of Mathematics, University of Pisa, Largo Bruno PonTECORVo 5, 56127 Pisa, Italy.

E-mail address: aleks.jevnikar@dm.unipi.it

Chang-Shou Lin, Taida Institute for Mathematical Sciences and Center for Advanced Study in Theoretical Sciences, National Taiwan University, Taipei, Taiwan.

E-mail address: cslin@math.ntu.edu.tw 